\title{
Prediction of NAFLD occurrence in prediabetes patients
}

\author{
COSMIN MIHAI VESA ${ }^{1}$, TAPAN BEHL ${ }^{2 *}$, SEBESTIAN NEMETH ${ }^{3}$, OVIDIU G. BRATU ${ }^{4}$, \\ CAMELIA C. DIACONU ${ }^{5}$, RADU DUMITRU MOLERIU ${ }^{6}$, NICOLETA NEGRUT ${ }^{7 *}$, DANA C. ZAHA ${ }^{1}$, \\ CRISTIANA BUSTEA $^{1 *}$, FLORENTINA IONITA RADU ${ }^{8}$ and SIMONA BUNGAU ${ }^{7}$ \\ ${ }^{1}$ Department of Preclinical Disciplines, Faculty of Medicine and Pharmacy, University of Oradea, \\ 410073 Oradea, Romania; ${ }^{2}$ Chitkara College of Pharmacy, Chitkara University, 140401 Punjab, India; \\ ${ }^{3}$ Department of Pharmacy, Faculty of Medicine and Pharmacy, University of Oradea, 410073 Oradea; \\ ${ }^{4}$ Clinical Department 3 and ${ }^{5}$ Department 5, 'Carol Davila' University of Medicine and Pharmacy, 050474 Bucharest; \\ ${ }^{6}$ Department of Mathematics, Faculty of Mathematics and Computer Science, West University of Timisoara, \\ 300223 Timisoara; ${ }^{7}$ Department of Psycho-Neuroscience and Recovery, Faculty of Medicine \\ and Pharmacy, University of Oradea, 410073 Oradea; ${ }^{8}$ Department of Gastroenterology, \\ Emergency University Central Military Hospital, 010825 Bucharest, Romania
}

Received July 8, 2020; Accepted August 7, 2020

DOI: $10.3892 /$ etm.2020.9320

\begin{abstract}
Non-alcoholic fatty liver disease (NAFLD) is a component of metabolic syndrome that significantly increases the cardiovascular risk of patients with glucose metabolism alterations. This study identified the prevalence of NAFLD, predictors of NAFLD and explored the link between insulin sensitivity, insulin resistance and leptinemia in 143 patients registered with prediabetes. Abdominal ultrasound was performed, and fasting insulin, postprandial insulin, leptin levels, common clinical/biochemical determinations were assessed. Certain variables that can predict NAFLD existence were determined and it was found that there is a high prevalence of NAFLD in patients with prediabetes. In univariate analysis, statistically significant associations $(\mathrm{P}<0.05)$ were found between waist circumference, systolic blood pressure, diastolic blood pressure, triglycerides, HDL-cholesterol, insulin sensitivity, $\beta$-cell function, leptin and NAFLD presence. The coefficients for the variables which obtain statistically significant association $(\mathrm{P}<0.05)$ are low, except for leptin which is the biochemical parameter that (in both univariate and multivariate analysis) is a strong predictor of NAFLD presence.
\end{abstract}

Correspondence to: Professor Ovidiu G. Bratu, Clinical Department 3, 'Carol Davila' University of Medicine and Pharmacy, 8 Eroii Sanitari Blvd., 050474 Bucharest, Romania

E-mail: ovi78doc@yahoo.com

Professor Florentina Ionita Radu, Department of Gastroenterology, Emergency University Central Military Hospital, 134 Calea Plevnei Street, 010825 Bucharest, Romania

E-mail: fionita04@yahoo.com

*Contributed equally

Key words: NAFLD, prediabetes patients, metabolic syndrome, leptin, HbA1c

\section{Introduction}

Non-alcoholic fatty liver disease (NAFLD) is the most frequent liver disease in developed countries and is usually associated with obesity (1). The presence of type 2 diabetes mellitus (T2DM) is associated with an increased prevalence of NAFLD of $\sim 50 \%$ (2). NAFLD is serious because it can progress to non-alcoholic steatohepatitis (NASH) and finally to cirrhosis, without the occurrence of any symptoms or modifications of the liver enzymes. The prevalence of liver fibrosis in patients with T2DM is estimated to be $7 \%$ (3). The presence of NAFLD increases the risk of developing diabetes $\sim 5$-fold (4). Insulin resistance is the main factor that leads to the incidence of NAFLD (5). Obesity leads to systemic insulin resistance that increases the circulating free fatty acid levels that will be stored in the liver, where they will also generate hepatic insulin resistance and activate inflammatory pathways, increase oxidative stress and promote the fibrosis process (5). Leptin is a hormone produced by adipose cells that should generate satiety; however, in the obese patients or with glucose metabolism deficiency there is also leptin resistance. It was demonstrated that excess triglycerides diminish the capacity of leptin to cross the blood-brain barrier (6).

Data regarding the association between NAFLD and prediabetes is less abundant in literature because prediabetes has been only recently recognized as a condition that has a high risk of metabolic complications. Studies revealed that individuals with prediabetes and NAFLD have a higher risk of progression to T2DM than individuals with prediabetes without NAFLD (7). The diagnosis of fatty liver is usually done with ultrasonography, although the most accurate method is liver biopsy (8). The study of the complex interactions between the presence of NAFLD and glucose metabolism alterations and their connective biomarkers resides in the fact that the associations of metabolic comorbidities have a significant effect on cardiovascular risk and overall mortality and morbidity $(9,10)$. 
Adipocytes produce leptin (a cytokine), having the function of regulating the body weight and energy balance, through its action on the hypothalamus (11), being involved in obesity. In the categories of patients susceptible to develop prediabetes or T2DM (obese and/or overweight patients), the serum levels of leptin are increased compared with normal-weight individuals and do not manage to reduce the drive to eat (12). Hyperleptinemia occurs as a consequence of leptin resistance either in the peripheral or in the central nervous system. The study of leptin interactions is particularly important in glucose metabolism alterations because there are complex relationships between insulin and leptin (11), relationships that undergo profound alterations in the context of increased adipose tissue, specific to prediabetes or type 2 diabetes patients.

The purpose of this stdy was to determine the prevalence of NAFLD in patients with prediabetes, to observe the clinical and biochemical differences between prediabetes patients with and without NAFLD, to identify those parameters that can predict the presence of NAFLD. The approach of this topic, the data provided, as well as the statistics, represent some of the strengths of this study.

\section{Patients and methods}

The study was conducted at the Clinical County Emergency Hospital of Oradea, Oradea, Romania, on 143 patients diagnosed with prediabetes, during one day of hospitalization. The 2-year period of study was between February 1st, 2018 and February 1st, 2020. The criteria for study participation were age 18-75 years and written consent for being included in the study, with a HbA1c level between 5.7-6.4\% (the reference values given by the current guidelines of American Diabetes Association (13) for prediabetes diagnosis). Exclusion criteria were: Patients under treatment with steroid drugs (known to increase glycaemia), patients with diabetes, patients with liver cirrhosis, patients suffering from alcoholism.

A total of 614 individuals were considered for diabetes suspicion during the specified interval. Two hundred and sixty-three were diagnosed with DM based on HbA1c $\geq 6.5$, 187 individuals without diabetes and/or prediabetes were excluded based on $\mathrm{HbA} 1 \mathrm{c}<5.7 \%$ and 164 patients were selected as potential candidates for our investigation (having a HbAlc between 5.7 and $6.4 \%$ ). Of the latter, after further applying the exclusion criteria, another 21 patients were excluded, thus 143 patients remained in the study. Patients included in the study were those who met the criteria for the prediabetes diagnosis and all the other criteria specified above, but who did not meet the criterion for diabetes diagnosis.

Individuals suspected of suffering from DM were evaluated during their hospitalization in the Clinical County Emergency Hospital of Oradea. Information and data regarding age, living environment (urban or rural), medical history, and medical treatment were collected. Systolic blood pressure (SBP), diastolic blood pressure (DBP), and waist circumference were measured, and body-mass index (BMI) was calculated. Standard biochemical blood tests were performed, including total cholesterol (TC), LDL-cholesterol (LDL-C), HDL-cholesterol (HDL-C), triglycerides (TG), and glycated hemoglobin. All subjects with confirmed prediabetes were asked to come for an evaluation of the presence of NAFLD via abdominal ultrasonography and blood sampling for further biochemical determinations: Serum glucose and serum insulin after a minimum of $8 \mathrm{~h}$ of fasting, serum glucose and serum insulin after $2 \mathrm{~h}$ from a glucose oral tolerance test and serum leptin. Serum insulin and leptin were tested in the accredited SC Biostandard 2007 SRL laboratory. NAFLD diagnosis was based on the presence of increased liver echogenicity, in the absence of alcohol intake. The diagnosis was made based on the evaluation of trained ultra-sonographers with the help of $3.5 \mathrm{MHz}$ linear transducer.

Insulinemia and leptinemia were also determined at the above laboratory using the immunochemical method with electrochemiluminescence detection (ECLIA); leptin was determined by ELISA method. Using the values of fasting glucose (G0), fasting insulin (INSO), post-prandial glucose (G120) and post-prandial insulin (INS120), the following indexes were determined:

- HOMA-IR, index for evaluation of insulin resistance: HOMA-IR=fasting insulin (INSO) $(\mu U / l) x$ fasting glucose (GLUO) $(\mathrm{mmol} / \mathrm{l}) / 22.5$

- HOMA\%B, index for evaluation of $\beta$ cell function: $H O M A \% B=20 x$ fasting insulin (INSO) $(\mu U / l) /($ fasting glucose (GLUO) (mmol/l)-3.5)

- HOMA\%S, insulin sensitivity index:

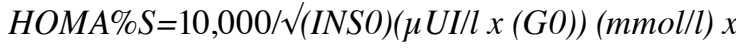
mean (G0-G120) x mean (INSO-INS120)

Leptin values were expressed as the logarithm 'log-leptin', since leptin is a variable with highly skewed distribution.

This study was conducted according to the WMA Declaration of Helsinki, Ethical Principles for Medical Research Involving Human Subjects; it was also approved by the Ethics Committee of the Clinical County Emergency Hospital of Oradea (Oradea, Romania). All subjects gave their written consent for the participation in the study.

The data were characterized by calculating the mean and standard deviation and by plotting the histograms of the main variables. As a first step, the variance of our data was tested using the Kolmogorov Smirnov test, when the sample was over 50 patients, and a Shapiro-Wilk test, when the samples was less than 50 patients. At $\mathrm{P}$-value $>0.05$, it was considered that there was a normal distribution, otherwise we assumed that the data were not normally distributed. A Student's t-test was used when comparing numerical variables with normal distribution, and a Mann-Whitney U test was used for numerical variables with skewed distribution. The confidence level was considered at $\alpha=0.05$ so P-value $<0.05$, indicated statistical significance. At the end of the cross-sectional study, a risk analysis was run, the odds ratio parameter was calculated, and the $95 \%$ confidence interval was estimated for the tested values. For the statistical significance a Chi-square test was applied, with $\alpha=0.05$ as confidence level. The statistical analysis was performed by using Biostat software, EPI INFOv7 and SPSSv17.

\section{Results}

From a total of 143 patients identified as suffering from prediabetes, $69(48.25 \%)$ were diagnosed with NAFLD and $74(51.75 \%)$ without NAFLD, according to their liver appearance using ultrasound. Compared with patients with prediabetes but 
Table I. Biochemical characteristics and analysis of the parameters of pre-diabetes patients without (n=74)/with (n=69) NAFLD.

\begin{tabular}{|c|c|c|c|}
\hline Parameter & Non NAFLD & NAFLD & P-value \\
\hline Age (years) & $44.8 \pm 9.47$ & $47.19 \pm 8.78$ & 0.21 \\
\hline $\operatorname{Sex}(\%$ men $)$ & 54.54 & 61.90 & 0.46 \\
\hline Impaired glucose tolerance $(\%)$ & 20.00 & 21.42 & 0.86 \\
\hline Impaired fasting glucose (\%) & 47.27 & 40.47 & 0.50 \\
\hline \multicolumn{4}{|l|}{ Impaired glucose tolerance +} \\
\hline Impaired fasting glucose (\%) & 32.20 & 38.09 & 0.55 \\
\hline Waist circumference $(\mathrm{cm})$ & $88.27 \pm 8.38$ & $93.30 \pm 7.23$ & $0.03^{\mathrm{a}}$ \\
\hline Hip circumference $(\mathrm{cm})$ & $94.46 \pm 8.16$ & $98.39 \pm 7.72$ & $0.04^{\mathrm{a}}$ \\
\hline Waist to hip ratio & $88.35 \pm 8.13$ & $93.20 \pm 7.24$ & $0.02^{\mathrm{a}}$ \\
\hline Body mass index $\left(\mathrm{kg} / \mathrm{m}^{2}\right)$ & $24.65 \pm 4.43$ & $26.04 \pm 3.56$ & 0.09 \\
\hline Systolic blood pressure (mm Hg) & $112.74 \pm 14.50$ & $131.45 \pm 33.02$ & $<0.01^{\mathrm{a}}$ \\
\hline Diastolic blood pressure (mm Hg) & $75.74 \pm 17.58$ & $88.97 \pm 23.62$ & $<0.01^{\mathrm{a}}$ \\
\hline Fasting serum glucose (mmol/l) & $5.92 \pm 0.47$ & $5.86 \pm 0.44$ & 0.52 \\
\hline Post-prandial serum glucose (mmol/l) & $7.76 \pm 1.58$ & $8.21 \pm 1.59$ & 0.16 \\
\hline Glycosylated haemoglobin (\%) & $5.85 \pm 0.65$ & $5.76 \pm 0.45$ & 0.44 \\
\hline Total cholesterol (mg/dl) & $182.49 \pm 37.36$ & $198.16 \pm 44.62$ & 0.06 \\
\hline Triglycerides (mg/dl) & $153.38 \pm 80.44$ & $209.73 \pm 137.18$ & $<0.01^{\mathrm{a}}$ \\
\hline HDL-cholesterol (mg/dl) & $38.49 \pm 7.58$ & $34.35 \pm 7.61$ & $<0.01^{\mathrm{a}}$ \\
\hline LDL-cholesterol (mg/dl) & $176.57 \pm 38.68$ & $182.18 \pm 45.56$ & 0.51 \\
\hline \multicolumn{4}{|l|}{ Glutamate pyruvate transaminase } \\
\hline$(\mathrm{GPT})(\mathrm{IU} / \mathrm{l})$ & $31.63 \pm 13.10$ & $38.80 \pm 18.43$ & $0.02^{\mathrm{a}}$ \\
\hline \multicolumn{4}{|l|}{ Gama-glutamate transaminase } \\
\hline$(\mathrm{GGT})(\mathrm{IU} / \mathrm{l})$ & $26.85 \pm 13.44$ & $33.57 \pm 12.76$ & $0.01^{\mathrm{a}}$ \\
\hline Alkaline phosphatase (IU/l) & $105.23 \pm 29.44$ & $108.07 \pm 23.93$ & 0.61 \\
\hline Glutamate oxaloacetate transaminase (GOT) (IU/l) & $26 \pm 8.91$ & $35.69 \pm 22$ & $<0.01^{\mathrm{a}}$ \\
\hline Fasting insulin $(\mu \mathrm{IU} / \mathrm{ml})$ & $13.4 \pm 3.38$ & $17.19 \pm 7.07$ & $<0.01^{\mathrm{a}}$ \\
\hline Postprandial insulin $(\mu \mathrm{IU} / \mathrm{ml})$ & $55.32 \pm 40.95$ & $74.02 \pm 38.52$ & $0.02^{\mathrm{a}}$ \\
\hline HOMA\%S & $58.90 \pm 21.55$ & $45.65 \pm 14.20$ & $<0.01^{\mathrm{a}}$ \\
\hline НОМА \% В & $130.14 \pm 34.86$ & $109.84 \pm 34.04$ & $<0.01^{\mathrm{a}}$ \\
\hline HOMA-IR & $3.52 \pm 0.93$ & $4.49 \pm 1.93$ & $<0.01^{\mathrm{a}}$ \\
\hline Log leptin (ng/ml) & $0.86 \pm 0.40$ & $1.28 \pm 0.18$ & $<0.01^{\mathrm{a}}$ \\
\hline
\end{tabular}

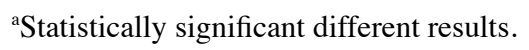

without NAFLD, patients with prediabetes and NAFLD had values that were statistically significant to a higher degree for waist circumference, SBP, DBP, TG, glutamic-pyruvic transaminase, glutamic oxaloacetic transaminase, gamma-glutamyl transferase, insulin resistance, and log-transformed leptin, and values that were statistically significant to a lower degree for HDL-C, $\beta$-cell function and insulin sensitivity. In order to obtain the P-value, Mann-Whitney test was applied for the numerical variables; for the qualitative variables chi square test for proportions was applied. The cut off point for significance was set at $\alpha=0.05$. The analysis and the obtained results are presented in Table I. Separately, an analysis was run on the two main groups (patients with/without NAFLD) and it was tested if the sex of the patient can be considered a determining factor in the dynamics of the parameters (Table I). P-value $<0.05$ was considered statistically significant.

These features are not significantly different between the 2 groups. Impaired glucose tolerance was identified in
$21.42 \%$ of patients with NAFLD and in $20 \%$ of patients without NAFLD; impaired fasting glucose was identified in $40.47 \%$ of patients with NAFLD and in $47.27 \%$ of patients without NAFLD; the association between impaired glucose tolerance and impaired fasting glucose was found in $38.09 \%$ patients with NAFLD and in $32.2 \%$ patients without NAFLD.

In univariate analysis, the factors that raised the risk of NAFLD presence in patients with prediabetes were: Increased waist circumference $(\mathrm{P}=0.005)$, increased systolic blood pressure $(\mathrm{P}=0.0003)$, increased diastolic blood pressure $(\mathrm{P}=0.0021)$, increased value of triglycerides $(\mathrm{P}=0.013)$, low values of HDL-cholesterol $(\mathrm{P}=0.0093)$, increased values of fasting insulin $(\mathrm{P}=0.0007)$, increased levels of postprandial insulin $(\mathrm{P}=0.0245)$, decreased level of insulin sensitivity $(\mathrm{P}=0.001)$, decreased $\beta$-cell function $(\mathrm{P}=0.0063)$, increased insulin resistance $(\mathrm{P}=0.0016)$ and increased values of leptin $(\mathrm{P} \leq 0.001)$. In the multivariate analysis, the factors that raised the risk of NAFLD presence in patients with prediabetes were as follows: Advanced age 
Table II. Predictor factors of NAFLD presence in patients with pre-diabetes.

\begin{tabular}{|c|c|c|c|c|c|c|}
\hline \multirow[b]{2}{*}{ Parameter } & \multicolumn{3}{|c|}{ Univariate analysis } & \multicolumn{3}{|c|}{ Multivariate analysis } \\
\hline & Coefficient & Standard error & P-value & Coefficient & Standard error & P-value \\
\hline Sex & -0.074 & 0.1026 & 0.4724 & -0.1799 & 0.0984 & 0.0716 \\
\hline Age & 0.007 & 0.0055 & 0.207 & 0.0100 & 0.0049 & 0.0425 \\
\hline WC & 0.0183 & 0.0059 & $0.0059^{\mathrm{a}}$ & -0.0113 & 0.0101 & 0.2688 \\
\hline BMI & 0.0203 & 0.0122 & 0.0993 & 0.0286 & 0.0200 & 0.1564 \\
\hline FBG & -0.0695 & 0.1103 & 0.5299 & -0.1288 & 0.2363 & 0.5873 \\
\hline 2-h PPG & 0.0447 & 0.0317 & 0.1615 & 0.0194 & 0.0282 & 0.4933 \\
\hline $\mathrm{HbA1c}$ & -0.0609 & 0.0883 & 0.4918 & -0.0761 & 0.0776 & 0.3297 \\
\hline SBP & 0.0069 & 0.0018 & $0.0003^{\mathrm{a}}$ & 0.0012 & 0.0023 & 0.6093 \\
\hline DBP & 0.0072 & 0.0023 & $0.0021^{\mathrm{a}}$ & 0.0003 & 0.0026 & 0.9235 \\
\hline $\mathrm{CHOL}$ & 0.0023 & 0.0012 & 0.0629 & 0.0019 & 0.0020 & 0.3489 \\
\hline Triglycerides & 0.0011 & 0.0004 & $0.013^{\mathrm{a}}$ & 0.0009 & 0.0007 & 0.2479 \\
\hline HDL-cholesterol & -0.0167 & 0.0063 & $0.0093^{\mathrm{a}}$ & -0.0095 & 0.0061 & 0.1220 \\
\hline LDL-cholesterol & 0.0008 & 0.0012 & 0.5231 & -0.0002 & 0.0017 & 0.9015 \\
\hline Fasting insulin & 0.03 & 0.0086 & $0.0007^{\mathrm{a}}$ & 0.0285 & 0.0982 & 0.7725 \\
\hline Postprandial insulin & 0.0028 & 0.0012 & $0.0245^{\mathrm{a}}$ & 0.0004 & 0.0014 & 0.7548 \\
\hline $\mathrm{HOMA} \% \mathrm{~S}$ & -0.0084 & 0.0025 & $0.001^{\mathrm{a}}$ & -0.0037 & 0.0031 & 0.2284 \\
\hline НОМА\%В & -0.0039 & 0.0014 & $0.0063^{\mathrm{a}}$ & -0.0040 & 0.0013 & $0.0022^{\circ}$ \\
\hline HOMA-IR & 0.1028 & 0.0317 & $0.0016^{\mathrm{a}}$ & -0.0550 & 0.3597 & 0.8788 \\
\hline Log leptin (ng/ml) & 0.6891 & 0.1116 & $\leq 0.001^{\mathrm{a}}$ & 0.5307 & 0.1312 & $0.0001^{\circ}$ \\
\hline
\end{tabular}

${ }^{\text {aS }}$ Statistically significant different results.

$(\mathrm{P}=0.0425)$, decreased $\beta$-cell function $(\mathrm{P}=0.0022)$ and increased values of leptin $(\mathrm{P}=0.0001)$ (Table II).

\section{Discussion}

In developed countries, NAFLD is currently known as the most common chronic liver disease, its prevalence being in constant growth all over the world as a result of the global obesity epidemic $(9,10,14)$. This pathology increases liver-related mortality and morbidity, as well the risk of some other comorbidities, namely type 2 diabetes and cardiovascular disease.

This study identified the prevalence of NAFLD, predictors of NAFLD and explored the link between insulin sensitivity, insulin resistance and leptinemia in people with prediabetes. To our knowledge, there are no previous studies in Romania on these aspects, in this population category, most studies being focused on people with diabetes, and the association between prediabetes and NAFLD is rarely explored even in the international literature.

The results obtained show that the prevalence of NAFLD was $48.25 \%$ in a population of patients with prediabetes. This prevalence is much higher than the $\sim 26 \%$ prevalence of NAFLD in the general population (15). Previous studies have identified models for predicting the existence of NAFLD in a patient, involving an equation that includes several of the patient's clinical and biochemical variables: Waist circumference, serum triglycerides, gamma-glutamyl transferase and body-mass index $(16,17)$. A positive result of this calculation or a fatty liver index $\geq 60$ indicate NAFLD presence (18).
Certain variables that can predict NAFLD existence in prediabetes patients were determined. In univariate analysis, statistically significant associations were found between: Waist circumference, systolic blood pressure, diastolic blood pressure, triglycerides, HDL-cholesterol, insulin sensitivity, insulin secretion, $\beta$-cell function, leptin and NAFLD presence. Table I shows that the coefficients for the variables which obtain statistically significant association $(\mathrm{P}<0.05)$ are low, except for leptin (a biochemical parameter which is a strong predictor of NAFLD presence, in both univariate and multivariate analysis) comparable to the results of other studies $(19,20)$.

The major quality of the study is that it demonstrates that the association of prediabetes and NAFLD is associated with a worse cardio-metabolic profile, a finding that is very useful in clinical practice; when the physician identifies a patient that fulfils the conditions of prediabetes diagnosis and also has NAFLD, it gives a valuable clue that the individual probably has a high aggregation of clinical (increased systolic or diastolic blood pressure) and preclinical (insulin resistance, high levels of circulating leptin) cardiovascular risk factors, and therefore will need to have a more aggressive approach in identifying those risk factors and managing them (21).

There is a high prevalence of NAFLD in patients with prediabetes. In univariate analysis, the factors that raised the risk of NAFLD presence in patients with prediabetes were as follows: Increased waist circumference, SBP, DBP, TG, insulin resistance, values of leptin, levels of fasting and postprandial insulin; low values of HDL-C; decreased level of insulin sensitivity and $\beta$-cell function. In the multivariate analysis, the 
factors that raised the risk of NAFLD presence in patients with prediabetes were advanced age, decreased $\beta$-cell function and increased values of leptin.

\section{Acknowledgements}

Not applicable.

\section{Funding}

No funding was received.

\section{Availability of data and materials}

Data are registered at the Clinical County Emergency Hospital of Oradea, Bihor County, Romania and SC BIOSTANDARD 2007 SRL, Oradea, Romania.

\section{Authors' contributions}

OGB, CCD, RDM, NN, DCZ, CB and FIR collected, analyzed and interpreted the patient data, regarding BMI determination and completion of questionnaires. CMV, TB, SN and SB made substantial contributions to the conception of the study and the interpretation of the data; also, they drafted the manuscript and were major contributors in writing the manuscript. All authors read and approved the final manuscript to be published. All the authors agreed to be accountable for all aspects of the work in ensuring that questions related to the accuracy or integrity of any part of the work are appropriately investigated and resolved.

\section{Ethics approval and consent to participate}

The research was conducted according to the WMA Declaration of Helsinki - Ethical Principles for Medical Research Involving Human Subjects. The study was also approved by the Ethics Committee of the Clinical County Emergency Hospital of Oradea (Oradea, Romania). All subjects gave their written consent for the participation in the study.

\section{Patient consent for publication}

Not applicable.

\section{Competing interests}

The authors declare that they have no competing interests.

\section{References}

1. Caldwell S and Argo C: The natural history of non-alcoholic fatty liver disease. Dig Dis 28: 162-168, 2010

2. Portillo-Sanchez P, Bril F, Maximos M, Lomonaco R, Bermacki D, Orsak B, Subbarayan S, Webb A, Hecht J and Cusi K: High prevalence of nonalcoholic fatty liver disease in patients with type 2 diabetes mellitus and normal plasma aminotransferase levels. J Clin Endocrinol Metab 100: 2231-2238, 2015.

3. Hazlehurst JM, Woods C, Marjot T, Cobbold JF and Tomlinson JW: Non-alcoholic fatty liver disease and diabetes. Metabolism 65: 1096-1108, 2016.
4. Shibata M, Kihara Y, Taguchi M, Tashiro M and Otsuki M: Nonalcoholic fatty liver disease is a risk factor for type 2 diabetes in middle-aged Japanese men. Diabetes Care 30: 2940-2944, 2007.

5. Chen Z, Yu R, Xiong Y, Du F and Zhu S: A vicious circle between insulin resistance and inflammation in nonalcoholic fatty liver disease. Lipids Health Dis 16: 203, 2017.

6. Veyrat-Durebex C, Poher AL, Caillon A, Somm E, Vallet P, Charnay Y and Rohner-Jeanrenaud F: Improved leptin sensitivity as a potential candidate responsible for the spontaneous food restriction of the Lou/C rat. PLoS One 8: e73452, 2013.

7. Nishi T, Babazono A, Maeda T, Imatoh T and Une H: Evaluation of the fatty liver index as a predictor for the development of diabetes among insurance beneficiaries with prediabetes. J Diabetes Investig 6: 309-316, 2014.

8. Schneider AL, Lazo M, Ndumele CE, Pankow JS, Coresh J, Clark JM and Selvin E: Liver enzymes, race, gender and diabetes risk, the atherosclerosis risk in communities (ARIC) study. Diabet Med 30: 926-933, 2013.

9. Remus Popa A, Fratila O, Rus M, Anca Corb Aron R, Vesa CM, Pantis C, Diaconu C, Bratu O, Bungau S and Nemeth S: Risk factors for adiposity in the urban population and influence on the prevalence of overweight and obesity. Exp Ther Med 20: 129-133, 2020.

10. Vesa CM, Popa L, Popa AR, Rus M, Zaha AA, Bungau S, Tit DM, Corb Aron RA and Zaha DC: Current data regarding the relationship between type 2 diabetes mellitus and cardiovascular risk factors. Diagnostics 10: 314, 2020.

11. Zhou Y and Rui L: Leptin signalling and leptin resistance. Front Med 7: 207-222, 2013.

12. Ernst MB, Wunderlich CM, Hess S, Paehler M, Mesaros A, Koralov SB, Kleinridders A, Husch A, Münzberg H, Hampel B, et al: Enhanced Stat 3 activation in POMC neurons provokes negative feedback inhibition of leptin and insulin signaling in obesity. J Neurosci 29: 11582-11593, 2009.

13. American Diabetes Association: 2. Classification and diagnosis of diabetes: Standards of medical care in diabetes-2020. Diabetes Care 43 (Suppl 1): S14-S31, 2020.

14. Abdel-Daim MM, El-Tawil OS, Bungau SG and Atanasov AG: Applications of antioxidants in metabolic disorders and degenerative diseases: Mechanistic approach. Oxid Med Cell Longev 2019: 4179676, 2019.

15. Pappachan JM, Babu S, Krishnan B and Ravindran NC: Non-alcoholic fatty liver disease: A clinical update. J Clin Transl Hepatol 5: 384-393, 2017.

16. Younossi ZM, Koenig AB, Abdelatif D, Fazel Y, Henry L and Wymer M: Global epidemiology of nonalcoholic fatty liver disease-meta-analytic assessment of prevalence, incidence, and outcomes. Hepatology 64: 73-84, 2016.

17. Balkau B, Lange C, Vol S, Fumeron F and Bonnet F; Group Study D.E.S.I.R: Nine-year incident diabetes is predicted by fatty liver indices: The French D.E.S.I.R. study. BMC Gastroenterol 10: 56, 2010.

18. Bedogni G, Bellentani S, Miglioli L, Masutti F, Passalacqua M, Castiglione A and Tiribelli C: The fatty liver index: A simple and accurate predictor of hepatic steatosis in the general population. BMC Gastroenterol 6: 33, 2006.

19. Cansanção K, Silva Monteiro L, Carvalho Leite N, Dávalos A, Tavares do Carmo MDG and Arantes Ferreira Peres W: Advanced liver fibrosis is independently associated with palmitic acid and insulin levels in patients with non-alcoholic fatty liver disease. Nutrients 10: 1586, 2018.

20. Denkmayr L, Feldman A, Stechemesser L, Eder SK, Zandanell S, Schranz M, Strasser M, Huber-Schönauer U, Buch S, Hampe J, et al: Lean patients with non-alcoholic fatty liver disease have a severe histological phenotype similar to obese patients. J Clin Med 7: 562, 2018.

21. Diaconu C, Salmen T, Gaman MA, Bratu OG, Mischianu D, Marcu RD, Suceveanu AI, Costache RS and Pantea Stoian A: SGLT2 inhibition in patients with type 2 diabetes and cardiovascular diseases: Which are the benefits? Rom J Mil Med 122: 16-21, 2019. 